\title{
Remarks on the Optimal Portfolio Problem in Discrete Variables with Multiple Stochastic Processes
}

\author{
Naohiro Yoshida and Naoyuki Ishimura
}

\begin{abstract}
We are concerned with the optimal portfolio problem under stochastic environment; in particular, we deal with the case of two independent stochastic processes in discrete variables. One process is typical random walk, which is regarded as a discrete version of the standard Brownian motion, and the other is the Poisson process. We derive a discrete Hamilton-Jacobi-Bellman (HJB) equation for the value function and try to solve it. Examples are also discussed.
\end{abstract}

Index Terms-Discrete hamilton-jacobi-bellman equation, discrete processes, multiple stochastic processes, optimal portfolio problem.

\section{INTRODUCTION}

The optimal portfolio problem under stochastic environment is an important theme for researches both from the theoretical and the practical point of view. Typical form of problems in continuous variables may be formulated as follows (observe Chapter 19 of [1]. See also [2]): Consider the consumer's investment and consumption problem over the given finite time horizon $T$. We wish to maximize

$$
E\left[\int_{0}^{T} F(t, c(t)) d t+\emptyset(X(T))\right]
$$

where the dynamics of the wealth $X(t)$ of the consumer at time $t$ is expressed as

$$
\begin{gathered}
d X(t)=X(t)(r(1-\theta)+\theta \alpha) d t-c(t) d t \\
+\theta \sigma X(t) d W(t) .
\end{gathered}
$$

Here $r$ is the risk free interest rate, $\alpha$ the drift coefficient of the asset price movement, and $\sigma$ is the volatility. $d W(t)$ stands for the standard Brownian motion. The control parameters are the consumption $c(t)$, which is assumed to be nonnegative, and $0 \leq \theta \leq 1 . F$ means the instantaneous utility function, whereas $\varnothing$ is also a utility function measuring the utility at the maturity $T$. In this setting, not only the terminal wealth but also the trajectory of the process is taken into account.

Typical procedure is that we then discuss the value function (compare Section 3 below), which characterize the

Manuscript received November 30, 2015; revised February 15, 2016. The second author is partially supported by Grant-in-Aid for Scientific Research (C) (Kakenhi) No. 15K04992 from Japan Society for the Promotion of Science (JSPS).

Naohiro Yoshida is with the Graduate School of Economics, Hitotsubashi University, Kunitachi, Tokyo 186-8601, Japan (e-mail: ed141003@g.hit-u.ac.jp).

Naoyuki Ishimura is with the Faculty of Commerce, Chuo University, Hachioji, Tokyo 192-0393, Japan (e-mail: naoyuki@tamacc.chuo-u.ac.jp). extreme value of the expectation above, and derive the Hamilton-Jacobi-Bellman (HJB) partial differential equation for this value function. However, HJB is known to be a challenging subject and in general it is hard to solve. Only that we can do is to presume the form of solution and manage to arrange necessary parameters.

Here, on the other hand, we are concerned with the optimal portfolio problem in discrete variables. Compared to the continuous case, it is expected that the problem may be easily computed. Indeed, we find the discrete analogue of the HJB equation and some examples show that solutions really exist and can be calculated. Observe the related work of [3] (see also [4]).

In addition, we are able to investigate the situation that two different stochastic processes are involved; one is the standard symmetric random walk, and the other is the Poisson process. For this situation also, it is possible to give a solution.

The paper is organized as follows. We recall some basic tools and provide a new formula for our discrete processes in Section II. Section III is devoted to our main problem and an example follows in Section IV. Section V concludes the article with discussions.

\section{BASIC TOOLS}

Here we recall our basic tools for analysis. The first part, which is concerned with a discrete analogue of the well-known Ito formula, while the second part, which is with the similar formula under multiple stochastic processes, seems new.

\section{A. Discrete Analogue of the Ito Formula}

Let $t=0,1,2, \cdots$ denote discrete time series and let $\{B(t)\}$ $(t=0,1,2, \cdots)$ with $B(0)=0$ be the standard symmetric random walks; that is,

$$
B(t)=\sum_{n=1}^{t} Z(n)
$$

where $\{Z(n)\}(n=1,2, \cdots)$ denote independent and identically distributed (i.i.d) random variables such that

$$
P(Z(n)=+1)=P(Z(n)=-1)=\frac{1}{2}, \quad n=1,2, \cdots .
$$

The process $\{B(t)\}(t=0,1,2, \cdots)$ with $B(0)=0$ may be regarded as a discrete version of the standard Brownian motion. Indeed, we have the next discrete analogue of the well-known Ito formula. See [4], [5] for the details.

Theorem 1. (a) For any function $f: Z \rightarrow R$, we have 


$$
\begin{aligned}
& f(B(t+1))-f(B(t)) \\
& =\frac{f(B(t)+1)-f(B(t)-1)}{2} Z(t+1) \\
& +\frac{f(B(t)+1)-2 f(B(t))+f(B(t)-1)}{2} .
\end{aligned}
$$

(b) For any $f: \boldsymbol{Z} \times \boldsymbol{N} \rightarrow \boldsymbol{R}$, we have

$$
\begin{aligned}
& f(B(t+1), t+1)-f(B(t), t) \\
& =\frac{f(B(t)+1, t+1)-f(B(t)-1, t+1)}{2} Z(t+1) \\
+ & \frac{f(B(t)+1, t+1)-2 f(B(t), t+1)+f(B(t)-1, t+1)}{2} \\
+ & f(B(t)+1, t+1)-f(B(t), t) .
\end{aligned}
$$

Sketch of Proof. We briefly provide a proof, which is elementary. Consider part (a). If $Z(t+1)=1$, then there holds $B(t+1)=B(t)+1$, and the right hand side agrees with the left hand side of the equation. If $Z(t+1)=-1$, then there holds $B(t+1)=B(t)-1$, and both side of the equation agrees. The proof of part (b) is similar. //

Next we turn our attention to the Poisson process $\{N(t)\}(t=0,1,2, \cdots)$ with $N(0)=0$ such that

$$
N(t)=\sum_{n=1}^{t} D(n)
$$

where

$$
D(n)=\left\{\begin{array}{l}
1 \text { with probability } q, \\
0 \text { with probability } 1-q .
\end{array}\right.
$$

For this process, we have the next formula.

Theorem 2. (a) For any function $f: Z \rightarrow R$, we have

$$
\begin{aligned}
f(N(t+1))-f & (N(t)) \\
& =(f(N(t)+1)-f(N(t))) D(t+1) .
\end{aligned}
$$

(b) For any $f: \boldsymbol{Z} \times \boldsymbol{N} \rightarrow \boldsymbol{R}$, we have

$$
\begin{aligned}
f(N(t+1), t+1) & -f(N(t), t) \\
= & (f(N(t)+1, t+1) \\
& -f(N(t), t+1)) D(t+1) \\
& +f(N(t), t+1)-f(N(t), t) .
\end{aligned}
$$

Sketch of Proof. We give a sketch of proof. Consider part (b). If $D(t+1)=1$, then there holds $N(t+1)=N(t)+1$.

If $D(t+1)=0$, then there holds $N(t+1)=$ $N(t)$. In any case, the right hand side agrees with the left hand side of the equation. //

\section{B. Underlying Price Process}

Now we introduce our underlying price process $\{X(t)\}(t=0,1,2, \cdots)$, which is assumed to be governed by the following stochastic equation in discrete variables.

$$
\begin{aligned}
& X(t+1)-X(t)=\mu+\sigma(B(t+1)-B(t)) \\
& \quad+\alpha(N(t+1)-N(t)),
\end{aligned}
$$

where $\{B(t)\} \quad(t=0,1,2, \cdots)$ with $B(0)=0$ and $\{N(t)\}(t=0,1,2, \cdots)$ with $N(0)=0$ are defined by (1) and (2), respectively and assumed to be independent. Here $\mu, \sigma$ and $\alpha$ are taken to be constants for simplicity. Below in Example, we treat the more general case.

Concerning this extended process (3), we are able to deduce the following difference formula, which seems to be new in the literature.

Theorem 3. (a) For any function $f: Z \rightarrow R$, we have

$$
\begin{gathered}
f(X(t+1))-f(X(t)) \\
=\frac{f(X(t)+\mu+\sigma)-f(X(t)+\mu-\sigma)}{2} Z(t+1) \\
+\frac{f(X(t)+\mu+\sigma)-2 f(X(t)+\mu)+f(X(t)+\mu-\sigma)}{2} \\
+f(X(t)+\mu)-f(X(t)) \\
+\frac{1}{2}\{f(X(t)+\mu+\sigma+\alpha)-f(X(t)+\mu+\sigma) \\
-f(X(t)+\mu-\sigma+\alpha) \\
+f(X(t)+\mu-\sigma)\} Z(t+1) D(t+1) \\
+\frac{1}{2}\{f(X(t)+\mu+\sigma+\alpha)-f(X(t)+\mu+\sigma) \\
+f(X(t)+\mu-\sigma+\alpha) \\
-f(X(t)+\mu-\sigma)\} D(t+1) .
\end{gathered}
$$

(b) For any $f: \boldsymbol{Z} \times \boldsymbol{N} \rightarrow \boldsymbol{R}$, we have

$$
\begin{gathered}
f(X(t+1), t+1)-f(X(t), t) \\
=\frac{f(X(t)+\mu+\sigma, t+1)-f(X(t)+\mu-\sigma, t+1)}{2} . \\
\cdot Z(t+1)+(f(X(t)+\mu, t+1)-f(X(t), t+1)) \\
+\frac{1}{2}\{f(X(t)+\mu+\sigma, t+1)-2 f(X(t)+\mu, t+1) \\
+f(X(t)+\mu-\sigma, t+1)\} \\
+(f(X(t), t+1)-f(X(t), t)) \\
+\frac{1}{2}\{f(X(t)+\mu+\sigma+\alpha, t+1)-f(X(t)+\mu+\sigma, t+1) \\
-f(X(t)+\mu-\sigma+\alpha, t+1) \\
+f(X(t)+\mu-\sigma, t+1)\} \\
\cdot Z(t+1) D(t+1) \\
+\sigma+\alpha, t+1)-f(X(t)+\mu+\sigma, t+1) \\
+f(X(t)+\mu-\sigma+\alpha, t+1) \\
-f(X(t)+\mu-\sigma, t+1)\} \cdot D(t+1) .
\end{gathered}
$$

The proof is also easy. We may safely omit the details.

For later purposes, we define the operator $L_{X} f(x, t)$ by taking the expectation of the right hand side of Theorem 3(b). That is

$$
\begin{aligned}
& L_{X} f(x, t): \\
& =f(x+\mu, t+1)-f(x, t) \\
& +\frac{f(x+\mu+\sigma, t+1)-2 f(x+\mu, t+1)+f(x+\mu-\sigma, t+1)}{2} \\
& +\frac{1}{2}\{f(x+\mu+\sigma+\alpha, t+1)-f(x+\mu+\sigma, t+1) \\
& +f(x+\mu-\sigma+\alpha, t+1)-f(X(t)+\mu-\sigma, t+1)\} \cdot q .
\end{aligned}
$$

It is to be noted that the difference operator $L_{X} f$ corresponds to the partial differential operator in the continuous processes.

\section{OPTIMAl PORTFOLIO PROBLEM}

Now we consider the optimal portfolio problem under discrete process (3). 
Suppose a controlled process $\{X(t)\}(t=0,1,2, \cdots, T)$ is given by

$$
\begin{aligned}
X(t+1)- & X(t) \\
& =\mu(u(t))+\sigma(u(t))(B(t+1)-B(t)) \\
& +\alpha(u(t))(N(t+1) \\
& -N(t))
\end{aligned}
$$

where $\{u(t)\}(t=0,1,2, \cdots, T)$ denotes a suitable adapted controlled process, and $T \in \boldsymbol{N}$ is the maturity date. We treat a simple case that constants parameter $\mu, \sigma$ and $\alpha$ in (3) are just replaced by controlled parameters $\mu(u), \sigma(u)$ and $\alpha(u)$. These are also can be generalized. Observe Example below.

Our aim is to determine the admissible control $\{u(t)\}(t=0,1,2, \cdots, T)$ which maximizes the expected utility at the maturity. To be precise, we want to solve the problem

$$
V(X(t), t):=\sup _{\{u(t)\}} E^{x, t}[U(X(t), T) \mid X(t)=x] .
$$

Here $U(x, t)$ stands for a utility function; namely, an increasing and strictly increasing function in $x$. We remark that $V(x, t)$ is called the value function.

Applying the well known Bellman principle, we obtain the discrete Hamilton-Jacobi-Bellman (HJB) equation as well as the verification theorem.

Theorem 4. (discrete Hamilton-Jacobi-Bellman (HJB) equation) For $t=0,1,2, \cdots, T$, we have

$$
\sup _{\{u(t)\}} L_{X} V(x, t)=0, \quad V(x, T)=U(x, T),
$$

where $L_{X}$ is defined in the end of Section 2.

Theorem 5. (Verification theorem) Let $W(x, t)$ solve the discrete HJB equation in Theorem 4 above; that is,

$$
\sup _{\{u(t)\}} L_{X} W(x, t)=0, \quad W(x, T)=U(x, T) .
$$

Then there holds

$W(x, t) \geq \sup _{\{u(t)\}} E^{x, t}[U(X(t), T) \mid X(t)=x]$, for any adapted process $\{u(t)\}$.

Moreover, there exists a suitable adapted process $\{u(t)\}$ such that $W(x, t)=V(x, t)$. [3].

The proof is straightforward and we may omit it here. See

In the next section we consider some examples to illustrate the validity of these theorems.

\section{EXAMPLE}

Here we provide one example to illustrate our theory.

We consider an optimal portfolio problem in the discrete Black-Scholes model with jumps. Let $S(t)$ and $R(t)$ be the underlying asset price process and risk-free asset price process respectively which are defined by

$$
\begin{gathered}
S(t+1)-S(t)=\mu S(t)+\sigma S(t) Z(t+1)+\alpha S(t) D(t \\
+1) \\
R(t+1)-R(t)=r R(t)
\end{gathered}
$$

where $\mu$ is an arbitrary constant, $r>0, \sigma>0$ and $\alpha>-1$. An agent invests at any time $t$ a proportion $u(t)$ of his wealth in $S(t)$ and $1-u(t)$ in $R(t)$. Then his wealth process $X(t)$ evolves according to

$$
\begin{aligned}
X(t+1)-X(t) & =\frac{u(t) X(t)}{S(t)}(S(t+1)-S(t)) \\
& +\frac{(1-u(t)) X(t)}{R(t)}(R(t+1)-R(t)) \\
& =(r+(\mu-r) u(t)) X(t) \\
& +\sigma u(t) X(t)(B(t+1)-B(t)) \\
& +\alpha u(t) X(t)(N(t+1)-N(t)) .
\end{aligned}
$$

That is, we take in (4)

$$
\begin{gathered}
\mu(u(t))=r+(\mu-r) u(t), \\
\sigma(u(t))=\sigma u(t) X(t), \\
\alpha(u(t))=\alpha u(t) X(t) .
\end{gathered}
$$

We assume that the agent have a utility function

$$
U(X(t), t)=\log X(t)
$$

Then the expected utility maximization problem is formulated as

$$
V(x, t)=\sup _{\{u(t)\}} E[U(X(T), T) \mid X(t)=x] .
$$

The corresponding discrete HJB equation becomes

$$
\begin{gathered}
\sup _{\{u(t)\}} L_{X} V(x, t)= \\
\sup _{\{u(t)\}}\left\{\frac{1-q}{2}(V((1+r+(\mu-r+\sigma) u(t)) x, t+1)\right. \\
+V((1+r+(\mu-r-\sigma) u(t)) x, t \\
+1)) \\
+\frac{q}{2}(V((1+r \\
+(\mu-r+\sigma+\alpha) u(t)) x, t+1) \\
+V((1+r+(\mu-r-\sigma+\alpha) u(t)) x, t \\
+1))-V(x, t)\}=0, \\
V(x, T)=\log x .
\end{gathered}
$$

We will seek a solution of the form

$$
V(x, t)=g(t)+\log x, \quad g(T)=0 .
$$

Substituting this into the above discrete HJB equation, we infer that

$$
\begin{aligned}
& \sup _{\{u(t)\}}\left\{\frac{1-q}{2} \log (1+r+(\mu-r+\sigma) u(t))(1+r\right. \\
+ & (\mu-r-\sigma) u(t)) \\
+ & \frac{q}{2} \log (1+r+(\mu-r+\sigma+\alpha) u(t))(1+r \\
+ & (\mu-r-\sigma+\alpha) u(t))+g(t+1)-g(t)\} \\
= & 0
\end{aligned}
$$

The maximization is attained by some constant $u^{*}$ that is obtained by solving 


$$
\begin{aligned}
& (1-q)\left(\frac{\mu-r+\sigma}{1+r+(\mu-r+\sigma) u^{*}}+\frac{\mu-r-\sigma}{1+r+(\mu-r-\sigma) u^{*}}\right) \\
& +q\left(\frac{\mu-r+\sigma+\alpha}{1+r+(\mu-r+\sigma+\alpha) u^{*}}\right. \\
& \left.+\frac{\mu-r-\sigma+\alpha}{1+r+(\mu-r-\sigma+\alpha) u^{*}}\right)=0 \text {. }
\end{aligned}
$$

In particular, when the process $S(t)$ is a discrete pure jump process which is the case where $\mu>0, \sigma=0$ and $-1<\alpha<$ 0 in the above model, we can find explicitly a solution to the problem easily. The optimal strategy $u^{*}$ is found to be

$$
u^{*}=-\frac{(1+r)(\mu-r+q \alpha)}{(\mu-r+\alpha)(\mu-r)}
$$

Therefore

$$
\begin{gathered}
g(t)=(T-t)\left((1-q) \log \frac{(1+r)(1-q) \alpha}{\mu-r+\alpha}\right. \\
\left.+q \log \frac{-(1+r) q \alpha}{\mu-r}\right),
\end{gathered}
$$

which gives the solution to (5).

\section{DISCUSSION}

In this article, we have dealt with the optimal portfolio problem in discrete variables with two different stochastic processes. Applying a discrete analogue of the Ito formula both for the symmetric random walk and the Poisson process, we have established a discrete Hamilton-Jacobi-Bellman (HJB) equation for the value function. Example shows that our method works well and characterize what is the critical solution. We do hope that our procedure can be extended to other types of problems. For instance, the modelling of the financial markets typically involves not necessarily independent several stochastic processes; the sudden jump in the price of stocks cannot be governed by the Brownian motion and thus Poisson like processes are indispensable. Our method may be applied to analyze such phenomena.

However, there remain several further topics for researches. One of them is the problem of convergence of our discrete processes under appropriate time scales. Indeed, if the process (3) involves only symmetric random walk, that is , if $\alpha=0$, then the change of time scales of the form below brings us to the ordinary Ito formula for standard Brownian motion: that is,

$X(t+\delta d t)-X(t)=\mu \delta d t+\sigma \sqrt{\delta d t}(B(t+\delta d t)-B(t))$
Then, taking the limit of $(B(t+\delta d t)-B(t)) / \sqrt{\delta d t}$ and invoking the central limit theorem, we are able to recover the usual Ito formula:

$$
\begin{gathered}
d f(X(t))=f^{\prime}(X(t)) \sigma d B(t)+f^{\prime}(X(t)) \mu d t \\
+\frac{1}{2} f^{\prime \prime}(X(t)) \sigma^{2} d t
\end{gathered}
$$

See [4] for the details.

Now we do want to make it clear if the Poisson process is present. That is, the convergence problem with the Poisson processe is certainly a theme for researches. We refer to [6] for relevant literature. The rigorous treatment is in progress now and we will return to this topic in the near future,

\section{ACKNOWLEDGMENT}

We are grateful to the referee for helpful suggestions, which helps in improving the manuscript.

\section{REFERENCES}

[1] T. Björk, Arbitrage Theory in Continuous Time, Oxford University Press, Oxford, 2004.

[2] R. Korn and E. Korn, "Option pricing and portfolio optimization," Graduate Studies in Mathematics 31, American Mathematical Society, Rhode Island, 2000.

[3] N. Ishimura and Y. Mita, "A note on the optimal portfolio problem in discrete processes," Kybernetika, vol. 45, pp. 681-688, 2009.

[4] T. Fujita, N. Ishimura, and N. Kawai, "Discrete stochastic calculus and its applications: An expository note," Advances Mathematical Economics, vol. 16, pp. 119-131, 2012

[5] T. Fujita, "Random walks and the stochastic analysis," Nihon Hyoron-shya, Tokyo, 2008.

[6] N. Yoshida, "Remarks on the transformation of Ito's formula for jump-diffusion processes," JSIAM Letters, vol. 7, pp. 29-32S, 2015.

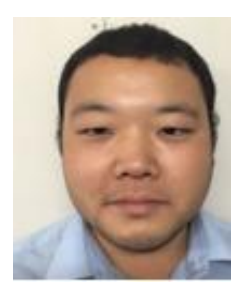

Naohiro Yoshida graduated from Faculty of Economics, Hitotsubashi University, Japan.

$\mathrm{He}$ is now a $\mathrm{PhD}$ course student at Graduate School of Economics, Hitotsubashi University, Japan.

Mr. Yoshida is a member of Japan Society of Industrial and Applied Mathematics (JSIAM).

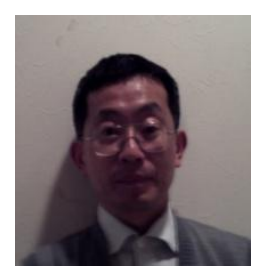

Naoyuki Ishimura graduated and obtained a PhD of mathematical sciences from the University of Tokyo, Japan.

$\mathrm{He}$ is now professor of mathematical finance at Faculty of Commerce, Chuo University, Tokyo, Japan. His current interest includes the mathematical finance and the theory of nonlinear partial differential equations.

Prof Ishimura is a member of Japan Society of Industrial and Applied Mathematics (JSIAM). 